\title{
THE EFFECT OF COULOMB INTERACTIONS ON THE HELIUM FLASH
}

\author{
A. Harpaz \\ Department of Physics, The Technion, Haifa, and \\ University of Haifa, School of Education of the Kibbutz Movement, Oranim \\ A. Kovetz \\ Department of Geophysics and Planetary Sciences \\ and School of Physics and Astronomy \\ Raymond and Beverly Sackler Faculty of Exact Sciences, Tel Aviv University
}

\begin{abstract}
Detailed evolutionary calculations show that Coulomb interactions between the charged particles of a stellar plasma reduce the core mass at which a low mass red giant undergoes the helium flash (contrary to a recent claim). This has implications for the determination of the rate of mass loss from red giants.
\end{abstract}

\section{INTRODUCTION}

The fact that Coulomb interactions between the charged particles of stellar plasmas result in a reduction of the pressure, at given density and temperature, has been known for a long time. Formulae for the pressure reduction in the case of a cold, dense, plasma were derived by Abrikosov (1960) and by Salpeter (1961), and the resulting reduction in the radii of white dwarfs was calculated by Hamada and Salpeter (1961). For the case of a finite temperature, several evaluations of the effect of Coulomb interactions on the equation of state were obtained in the 1970's (e.g., Grossman and Graboske, 1971; Shaviv and Kovetz, 1972; Hansen, 1973; Fontaine, Graboske and van Horn, 1977). The effect has been included in some calculations of stellar evolution, mainly cooling sequences of white dwarfs and low-mass main sequence stars (e.g., Lamb and van Horn, 1975; Shaviv and Kovetz, 1976; Iben and Tutukov, 1984; Vandenberg, Hartwick and Dawson, 1983).

Since the Coulomb correction is generally small (for example, Hamada-Salpeter white dwarf radii are typically about 7\% less than Chandrasekhar's), its inclusion in stellar evolution calculations is not expected to lead to any dramatic effect. But there are some cases, besides white dwarfs and M-dwarfs, in which the effect is worth considering. One of these is the onset of the helium flash in the cores of red giants, which we consider in Section II. Others will be mentioned in the discussion (Section III).

\section{THE EFFECT OF COULOMB CORRECTIONS ON THE HELIUM FLASH}

While a low mass star is ascending the red giant branch (RGB), its growing helium core is continually contracting. This leads to higher and higher core temperatures (the so-called gravitational energy source). 
Neutrino emission, mainly at the center, and radiative heat conduction lead to a temperature profile with an off-center maximum. When the core becomes sufficiently massive, the energy production due to helium burning exceeds the losses, and since the matter is degenerate, a flash occurs.

It is easy to determine the effect of the Coulomb correction on the core mass at the onset of the flash. If we imagine the Coulomb interaction being turned on at any instant, the pressure reduction will lead to sudden core contraction, and hence to higher temperature. It could trigger the flash in a core that would otherwise still need to grow somewhat. But since the processes leading to the flash proceed at a finite rate, and the Coulomb correction depends on density and temperature at each stage, a precise evaluation of the effect requires that it be turned on at an early stage. In order to demonstrate the effect, we have therefore carried out two parallel computations, with and without the correction.

We have evolved two $0.9 \mathrm{M}_{\odot}$ population II models $(\mathrm{X}=0.7, \mathrm{Z}=0.001)$ through the main sequence and the RGB. In the first one (Model $A$ ) we used the Shaviv-Kovetz equation of state, which includes the Coulomb correction, and allowed for mass loss according to the Reimers formula

$$
\dot{\mathrm{M}}=-410^{-13} \eta \mathrm{LR} / \mathrm{M} \quad \mathrm{M}_{\odot} / \mathrm{y}
$$

with $\eta=1$. (For other details regarding the method and the input physics, see Harpaz, Kovetz and Shaviv, 1987). When this model formed a core of $0.4 \mathrm{M}_{\odot}, 7.15410^{9}$ years from the ZAMS, its total mass was $\mathrm{M}=0.769 \mathrm{M}_{\odot}$, its central temperature was $\mathrm{T}_{c}=74.510^{6} \mathrm{~K}$, but helium burning still provided less energy $\left(L_{\mathrm{He}}\right)$ than was carried off by neutrinos $\left(L_{\nu}\right)$. The Coulomb parameter (ratio of $\left\langle\mathrm{Z}^{2}\right\rangle \mathrm{e}^{2} /\langle\mathrm{r}\rangle$ to $\mathrm{kT}$ ) was $\Gamma_{\mathrm{c}}=0.62$ at the center.

The second model (B) was evolved, again through the main sequence and the RGB, with the only difference that the Coulomb correction was switched off. Its evolution was somewhat slower than that of model A. For example, it took $7.32910^{9}$ years (from the ZAMS) to form a helium core of $0.4 \mathrm{M}_{\odot}$. Its total mass, central temperature and central Coulomb parameter at this stage were $M=0.7743 \mathrm{M}_{\odot}$, $\mathrm{T}_{\mathrm{c}}=73.310^{6} \mathrm{~K}$ and $\Gamma_{\mathrm{c}}=0.64$.
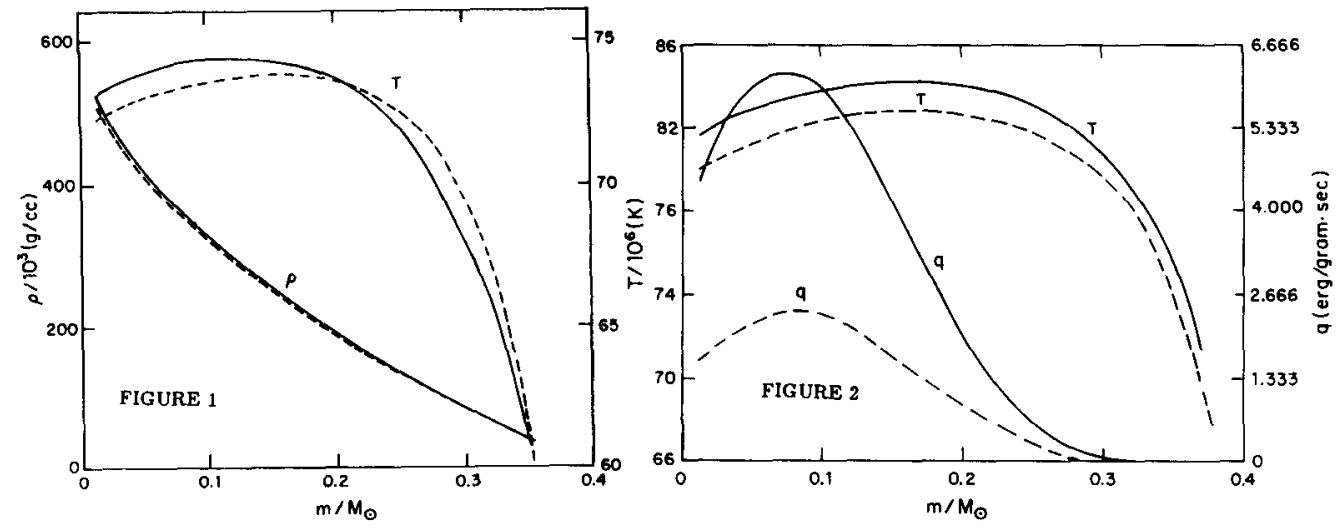

Figure 1 shows the density and temperature profiles in the central regions of models $\mathrm{A}$ and $\mathrm{B}$ when each one had a core of $0.40 \mathrm{M}_{\odot}$, and Figure 2 shows the temperature and nuclear production rate when each of the models has formed a core of $0.43 \mathrm{M}_{\odot}$. At this stage, $\mathrm{L}_{\mathrm{He}}$ just exceeded $\mathrm{L}_{\nu}$ for model $\mathrm{A}$, but was still less than $L_{\nu}$ in model $B$. In all figures, solid lines apply to model $A$, and dashed lines to model B. 


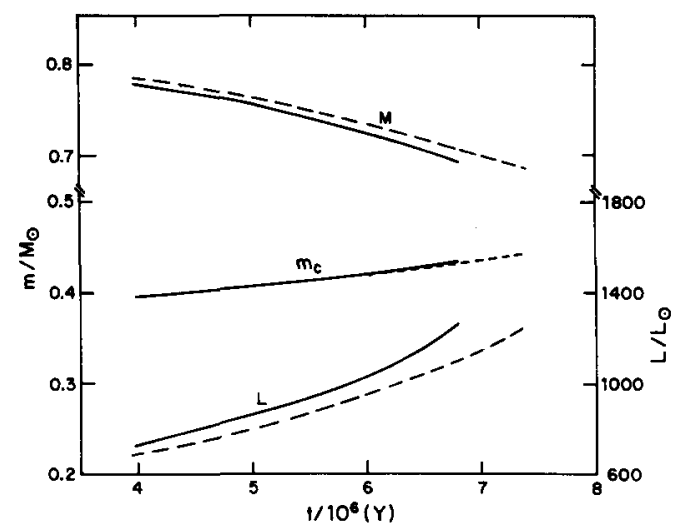

FIGURE 3

The helium burning in model $A$ became a runaway at $M_{c}=0.435 \mathrm{M}_{\odot}, M=0.693 \mathrm{M}_{\odot}$, and its $\mathrm{RGB}$ evolution came to an end. Model $B$ continued its evolution until it reached $M_{c}=0.440 M_{\odot}$, with $\mathrm{M}=0.690 \mathrm{M}_{\odot}$. Figure 3 shows the parallel evolution, represented by the total mass, the core mass and the luminosity, of the two models. Time for each model has been reset to zero when the core mass reached $0.175 \mathrm{M}_{\odot}$ (although the two models were not of the same age at this point).

\section{DISCUSSION}

The considerations of the preceding section have shown, and the results of two parallel calculations have demonstrated, that the Coulomb correction hastens the onset of the helium flash in low mass stars. These stars will therefore spend less time on the RGB, will lose a smaller amount of mass, and will leave the RGB at a smaller luminosity. They will arrive at the horizontal branch with smaller cores and more massive envelopes, and at an earlier time. The differences are all quite small, of the order of a few per cent (like the Hamada-Salpeter results for white dwarf radii), because the equation of state is dominated by the degenerate electrons.

We note that Mazzitelli and D'Antona, who included the Coulomb correction in an evolutionary calculation of a $1.0 \mathrm{M}_{\odot}$ population I model, obtained a higher (by about $0.05 \mathrm{M}_{\odot}$ ), rather than lower, core mass at the helium flash than did Sweigart and Gross (1978), who did not include the correction. Of course, small differences between results obtained with different computer codes, using different methods and different input physics, need not necessarily be due to a single correction in the equation of state. (Our Model B flash core mass happens to differ from that of Sweigart and Gross by less than $0.03 \mathrm{M}_{\odot}$ ). We are certainly not in a position to analyze differences between the results of Mazzitelii and D'Antona and those of Sweigart and Gross. But Mazzitelli and $\mathrm{D}^{\prime}$ Antona claim that the correction should indeed lead to a higher core mass at helium flash: "At the flash conditions (typically $\mathrm{T}=10^{8} \mathrm{~K}$ and $\rho=10^{6}$ $\mathrm{gcm}^{-3}$, at the ratio $\Gamma$ of Coulombian to thermal energy is $\sim 0.5$, and the excess internal energy due to Coulombian effects can be 15\%-20\%; Hansen (1973). This means that our models which take into account Coulombian corrections need to contract more and thus reach larger total core masses before the flash may be ignited". Of course, at given $T$ and $\rho$, the "excess internal energy" is negative, and if we understand correctly, what Mazzitelli and $D^{\prime}$ Antona are saying is that Coulomb interactions make the 
matter colder, and therefore delay the onset of the flash. But the core of a red giant behaves pretty much like a star, which becomes hotter when it loses energy. This is essentially the argument we have used at the beginning of Section II.

Renzini $(1977,1981)$ used the results of evolutionary calculations carried out by Rood (1972), Tarbell and Rood (1975) and by Sweigart and Gross (1978) to obtain an upper limit on the parameter $\eta$ in the mass loss formula (1). As he remarked, the very existence of horizontal and asymptotic giant stars in globular clusters proves that mass loss does not completely strip the RGB stars of their envelopes. This, he showed, meant that $\eta$ could not be larger than about 0.6. But a detailed analysis of globular cluster HR-diagrams led him to place more stringent constraints on the envelope masses of horizontal branch stars. In this way he was able to show that $\eta$ should not exceed $0.4 \pm 30 \%$. Since the Coulomb correction (which had not been included in the calculations on which Renzini based his analysis) leads to higher total masses, and especially to higher envelope masses $(0.258$ in our Model A vs. 0.250 in our Model B), Renzini's method should lead to a slightly higher upper limit on $\eta$.

In conclusion, we should perhaps mention that there are other advanced stages in stellar evolution, in which the Coulomb correction may be worth considering. One is the onset of carbon burning, where changes similar to those we have considered may be expected. Another is the luminosity-core mass relation for thermally pulsating asymptotic giant branch stars.

\section{REFERENCES}

Abrikosov, A. A., 1960, Sov. Phys. JETP, 12, 1254.

Fontaine, G., Graboske, H. C. and van Horn, H. M., 1977, Ap. J. Suppl., 35, 293.

Grossman, A. S. and Graboske, H. C., 1971, Ap. J. 164, 475.

Hamada, T. and Salpeter, E. E., 1961, Ap. J., 134, 683.

Hansen, J.P., 1973, Phys. Rev. A., 8, 3096.

Harpaz, A., Kovetz, A. and Shaviv, G., 1987, Ap. J., in press.

Iben, I. and Tutukov, A. V., 1984, Ap. J., 282, 615.

Lamb, D. Q. and van Horn, H.M., 1975, Ap. J., 200, 306.

Mazzitelli, I. and D'Antona, F., 1986, Ap. J., 311, 762.

Reimers, D., 1975, Mem. Soc. Roy. Sci., Liege, 6e Ser., 8, 369.

Renzini, A., 1977, Advanced Stages in Stellar Evolution, Ed. I. Iben, A. Renzini and D. N. Schramm, Geneva Observatory, Switzerland.

Renzini A., 1981, Effects of Mass Loss on Stellar Evolution, Ed. C. Chiosi and R. Stalio, Reidel Publishing Co., Dordrecht, Holland.

Rood, R. T., 1972, Ap. J., 177, 681.

Salpeter, E. E., 1961, Ap. J., 134, 669.

Shaviv, G. and Kovetz, A., 1972, Astron. and Astrophys., 16, 72.

Shaviv, G. and Kovetz, A., 1976, Astron. and Astrophys., 51, 383.

Sweigart, A. V. and Gross, P. G., 1978, Ap. J. Suppl., 36, 405.

Tarbell, T. D. and Rood, R. T., 1975, Ap. J., 199, 443.

Vandenberg, D. A., Hartwick, F. D. A. and Dawson, P., 1983, Ap. J., 266, 747. 